Revista Brasileira de Agricultura Irrigada v.9, nº.3, p. 102 - 112, 2015

ISSN 1982-7679 (On-line)

Fortaleza, CE, INOVAGRI - http://www.inovagri.org.br

DOI: $10.7127 /$ rbai.v9n300276

Protocolo 276.15 - 05/01/2015 Aprovado em 08/05/2015

\title{
INFLUÊNCIA DA DIFUSIVIDADE E CONDUTIVIDADE HIDRÁULICA NA INFILTRAÇÃO DE ÁGUA EM UM LATOSSOLO VERMELHO SOB DIFERENTES SISTEMAS DE CULTIVO
}

\author{
Fernando Nobre Cunha ${ }^{1}$, Nelmício Furtado da Silva ${ }^{2}$, Luciana Minervina de Freitas Moura ${ }^{3}$, \\ Marconi Batista Teixeira ${ }^{4}$ José Joaquim de Carvalho ${ }^{5}$, Rogerio Teixeira da Silva ${ }^{6}$
}

\section{RESUMO}

Objetivou-se com este estudo avaliar a difusividade e a condutividade hidráulica saturada e não saturada em um Latossolo Vermelho Distroférrico e o seu impacto na infiltração de água no sistema de plantio convencional e direto com a cultura do milho. O estudo foi realizado em áreas localizadas no Instituto Federal Goiano - Câmpus Rio Verde, GO. Tratamentos: 1) Sistema plantio convencional; 2) Sistema de plantio direto. O delineamento experimental foi inteiramente casualizado com oito repetições. A metodologia utilizada foi a do infiltrômetro de anéis concêntricos. $\mathrm{O}$ ajuste dos parâmetros das equações que representa o modelo para o solo foi realizado através do aplicativo RETC versão 6.02. Foi considerado o conteúdo de água, capacidade hidráulica, condutividade hidráulica de solo não saturado, efetivo conteúdo de água e difusividade versus o logaritmo da carga de pressão. A condutividade hidráulica no sistema de plantio direto foi 7,2\% maior do que a observada no sistema de plantio convencional. A capacidade hidráulica mostrou-se mais elevada no sistema de plantio convencional sendo esta 9,1\% superior a encontrada no sistema plantio direto. A difusividade hidráulica inicial no sistema de plantio direto foi $16 \%$ superior ao sistema de plantio conveniconal.

Palavras-chave: plantio convencional, plantio direto, infiltrômetro de anéis.

\footnotetext{
${ }^{1}$ Eng. Agrônomo, Doutorando em Ciências Agrárias - Agronomia, Laboratório de Hidráulica e Irrigação do Instituto Federal Goiano - Câmpus Rio Verde, Rodovia Sul Goiana, Km 01, CEP: 75.901-170, Rio Verde - GO, e-mail: fernandonobrecunha@ hotmail.com

${ }^{2}$ Eng. Agrônomo, Doutorando em Ciências Agrárias - Agronomia, Laboratório de Hidráulica e Irrigação do Instituto Federal Goiano - Câmpus Rio Verde, Rio Verde - GO, e-mail: nelmiciofurtado@gmail.com

${ }^{3}$ Eng. Agrônoma, Mestranda em Ciências Agrárias - Agronomia, IFGoiano - Câmpus Rio Verde, Rio Verde GO, e-mail: lucianaminervina@gmail.com

${ }^{4}$ Eng. Agrônomo, Prof. Dr. em Agronomia, Laboratório de Hidráulica e Irrigação do IFGoiano - Câmpus Rio Verde, Rio Verde - GO, e-mail: marconibt@gmail.com

5 Tecnólogo em Irrigação e Drenagem, Pós-Doutorando em Ciências Agrárias - Agronomia, Laboratório de Hidráulica e Irrigação do IFGoiano - Câmpus Rio Verde, Rio Verde - GO, e-mail: josejoaquimcarvalho@yahoo.com.br

6 Eng. Agrícola, Prof. Dr. Centro de Universitário Adventista de São Paulo - UNASP. Rogerio.teixeira.silva@hotmail.com
} 


\title{
INFLUENCE OF HYDRAULIC CONDUCTIVITY AND DIFFUSIVITY IN WATER INFILTRATION ON A OXISOL UNDER DIFFERENT CROPPING SYSTEMS
}

\begin{abstract}
The objective of this study was to evaluate the diffusivity and the saturated and unsaturated hydraulic conductivity in a Oxisol and its impact on water infiltration in the conventional tillage system and direct cultivated with corn.. The study was conducted in areas located at the Federal Institute Goiano - Campus Rio Verde, GO. Treatments: 1) conventional tillage system; 2) no-till system. The experimental design was completely randomized with eight repetitions. The methodology used was the infiltrometer of concentric rings. The setting of the parameters of the equations representing the model for the soil was performed using the RETC version 6.02 application. Was regarded as the water content, hydraulic capacity hydraulic conductivity unsaturated soil, water content and effective diffusivity versus the logarithm of the pressure load. The hydraulic conductivity in no-tillage system was $7.2 \%$ higher than that observed in the conventional tillage system; The hydraulic capacity was found to be higher in conventional tillage which is $9.1 \%$ higher than that found in no-tillage; The initial hydraulic diffusivity in no-tillage system was $16 \%$ higher than conveniconal planting system.
\end{abstract}

Keywords: conventional planting, tillage, infiltrometer rings.

\section{INTRODUÇÃO}

Os sistemas de plantio direto e convencional afetam as propriedades físicohídricas do solo, inclusive parâmetros como a difusividade e condutividade hidráulica do solo saturado e não saturado, influenciando a sua capacidade de retenção e disponibilidade de água (SILVA et al., 2012).

A eficiência do manejo do solo e da água estão condicionadas ao conhecimento do processo de infiltração da água e sua relação com as propriedades do solo, pois a infiltração é determinante para o balanço hídrico na região do sistema radicular (REICHARDT \& TIMM, 2012). A infiltração consiste no processo de entrada da água no perfil do solo (SOBRINHO et al., 2003). Com um valor inicialmente alto, a infiltração decresce após determinado tempo tornando-se constante com a saturação do solo, podendo ser chamada de velocidade de infiltração básica (BERNARDO et al., 2006; CUNHA et al., 2009).

A água do solo não é estática, mas dinâmica, movimentando-se em função do gradiente de seu potencial entre dois pontos quaisquer do solo. Atualmente, não mais se procura classificar a água do solo, mas sim caracterizá-la quantitativamente, para fins de estudo de sua disponibilidade, para as plantas, durante a sua movimentação pelo solo (BERNARDO et al., 2006).

O fluxo de água no solo, saturado e não saturado, é expresso pela condutividade hidráulica, que depende da estrutura e do conteúdo de água no solo (GONÇALVES \& LIBARDI, 2013). A difusividade hidráulica é outro parâmetro hídrico determinante na previsão da absorção de água pela planta e que está relacionada a evaporação de água (FEDDES et al., 1976). A difusividade referese à facilidade com que a umidade se expande no espaço e no tempo, ou seja, para o fluxo horizontal o gradiente de potencial total de água no solo é igual ao gradiente de potencial matricial, dado que a componente gravitacional é constante (CONCEIÇÃO, 2013).

Em solos com baixa condutividade hidráulica, a água disponível é insuficiente para suprir as necessidades hídricas da planta, levando-a ao murchamento (SILVA, 2005). Consequentemente, a produtividade da cultura 
é afetada devido ao impacto negativo substancial no crescimento e desenvolvimento da planta decorrente da redução no fluxo xilemático (LAWLOR \& CORNIC, 2002).

$\mathrm{O}$ conhecimento do movimento da água no solo é de extrema importância para a produção agrícola, pois interfere na irrigação, drenagem, armazenamento e transporte de água e de nutrientes, na infiltração da água no solo e no escoamento superficial (MELO \& BARROS, 2007), sendo determinantes para o adequado manejo do solo e da cultura (BRITO et al., 2009). Para Silva et al. (2014) a adoção de sistemas de manejo que mantém ou melhoram a qualidade do solo tem-se apresentado como uma alternativa viável no sistema produtivo agrícola.

Diante disso, objetivou-se com este estudo avaliar a difusividade e a condutividade hidráulica saturada e não saturada e o seu impacto na infiltração de água nos sistemas de plantio convencional e direto em um Latossolo Vermelho distroférrico.

\section{MATERIAL E MÉTODOS}

$\mathrm{O}$ estudo foi realizado em áreas localizadas no Instituto Federal Goiano Câmpus Rio Verde, GO, em 2014 (17²8'28" S e 5053'57" O, altitude média de 720 metros e relevo suave ondulado - $6 \%$ de declividade). $\mathrm{O}$ clima da região é classificado conforme Köppen, como Aw (tropical), com chuva nos meses de outubro a maio, e com seca de junho a setembro. A temperatura média anual é de 20 a $35{ }^{\circ} \mathrm{C}$ e as precipitações variam de 1.500 a $1.800 \mathrm{~mm}$ anuais. $\mathrm{O}$ solo predominante das áreas de estudo é classificado como Latossolo Vermelho distroférrico (LVdf) de textura média (EMBRAPA, 2006).

Em cada área experimental foi demarcada uma parcela, onde foram realizados os ensaios de campo para avaliação dos atributos submetidos a diferentes manejos. Assim foram selecionados dois tratamentos, a saber: 1) Sistema plantio convencional; 2) Sistema de plantio direto; ambos cultivado com a cultura do milho. O delineamento experimental foi inteiramente casualizado com oito repetições.

Os testes de infiltração de água no solo foram realizados em cada um dos locais de estudo. A metodologia utilizada foi a do infiltrômetro de anéis concêntricos, de acordo com Bernardo (2006), constituído de um anel externo com $0,20 \mathrm{~m}$ de diâmetro e $0,40 \mathrm{~m}$ de altura e um anel interno com $0,10 \mathrm{~m}$ de diâmetro e $0,40 \mathrm{~m}$ de altura. $\mathrm{O}$ cilindro foi inserido no solo a uma profundidade de aproximadamente $0,20 \mathrm{~m}$, com uma régua fixada na borda do cilindro interno, projetada até tocar a superfície do solo, para medir a lâmina de água.

Os tempos em minutos de cada leitura foram: $0,1,2,5,10,15$, e 30 minutos a iniciar do instante zero, e com repetições a cada 30 minutos até o tempo total de duração de cada teste de 210 minutos.

Os testes foram realizados até que a taxa de infiltração, observada no anel interno, tornase aproximadamente constante com o tempo. $\mathrm{O}$ critério adotado neste trabalho para condição de taxa de infiltração constante foi quando o valor de leitura da carga de água no cilindro interno se repetiu pelo menos três vezes.

A condutividade hidráulica do solo saturado $\left(\mathrm{K}_{\mathrm{s}}\right)$ foi determinada através da seguinte equação (BERNARDO et al., 2006):

$$
\mathrm{K}_{\mathrm{S}}=\frac{\mathrm{QxL}}{\mathrm{A}(\mathrm{L}+\mathrm{h})}
$$

em que:

$\mathrm{K}_{\mathrm{s}}$ - Condutividade hidráulica do solo saturada $\left(\mathrm{cm} \mathrm{h}^{-1}\right)$;

L - Profundidade do anel enterrado $(\mathrm{cm})$;

$\mathrm{Q}$ - vazão percolada através da amostra $\left(\mathrm{cm}^{3}\right.$ $\left.\mathrm{h}^{-1}\right)$;

A - seção transversal da amostra $\left(\mathrm{cm}^{2}\right)$;

h - carga hidráulica $(\mathrm{cm})$. 
Após realização das análises, a curva característica de água no solo foi obtida, ajustando-se o conteúdo de água no solo $(\theta)$ no eixo das ordenadas (escala decimal) em função da tensão de água no solo $\left(\psi_{\mathrm{m}}\right)$ correspondente ao eixo das abscissas (escala logarítmica), ajustando-se a equação de van Genuchten (1980) utilizando o programa RETEC v. 6.02 (van GENUCHTEN, 2009). Segundo o modelo original de van Genuchten-Mualem, estas relações foram definidas analiticamente da seguinte forma:

$$
\begin{array}{ll}
\theta(\mathrm{h})=\theta_{\mathrm{r}}+\frac{\theta_{\mathrm{s}}-\theta_{\mathrm{r}}}{\left(1+|\alpha \mathrm{h}|^{\mathrm{n}}\right)^{\mathrm{m}}} & \mathrm{h}<0 \\
\theta(\mathrm{h})=\theta_{\mathrm{s}} & \mathrm{h} \geq 0
\end{array}
$$

em que:

$\theta$ - conteúdo volumétrico de água $\left(\mathrm{cm}^{3} \mathrm{~cm}^{-3}\right)$;

$\mathrm{h}$ - potencial matricial $(\mathrm{cm})$;

$\theta_{\mathrm{r}}-$ umidade residual $\left(\mathrm{cm}^{3} \mathrm{~cm}^{-3}\right)$;

$\theta_{\mathrm{s}}$ - umidade saturação, respectivamente $\left(\mathrm{cm}^{3}\right.$ $\left.\mathrm{cm}^{-3}\right)$;

$\alpha$ - parâmetro relacionado com o inverso da pressão de entrada de $\operatorname{ar}\left(>0, \mathrm{~cm}^{-1}\right)$;

$\mathrm{n}(>1)$ e $\mathrm{m}=1-1 / \mathrm{n}$ (MUALEM, 1976): parâmetros empíricos adimensionais;

Van GENUCHTEN propôs a seguinte equação analítica para determinação da condutividade hidráulica não saturada $(K)$ de um solo em função do teor de umidade volumétrico:

$$
\mathrm{K}=\mathrm{K}_{\mathrm{S}}\left(\frac{\theta-\theta_{\mathrm{r}}}{\left(\theta_{\mathrm{s}}-\theta_{\mathrm{r}}\right)}\right)^{\frac{1}{2}}\left(1-\left[1-\left(\frac{\theta-\theta_{\mathrm{r}}}{\left(\theta_{\mathrm{s}}-\theta_{\mathrm{r}}\right)}\right)^{\frac{1}{\mathrm{~m}}}\right]^{\mathrm{m}}\right)^{2}
$$

em que:

$\mathrm{k}$-condutividade hidráulica não saturada (L $\mathrm{T}^{-1}$ );

$\mathrm{k}_{\mathrm{s}}$ - condutividade hidráulica na condição saturada $\left(\mathrm{L} \mathrm{T}^{-1}\right)$; $\theta_{\mathrm{s}}$ - é o teor de umidade volumétrico de saturação, em $\left[\mathrm{L}^{3} \mathrm{~L}^{-3}\right]$;

$\theta_{\mathrm{r}}$ - é o teor de umidade volumétrico residual, em $\left[\mathrm{L}^{3} \mathrm{~L}^{-3}\right]$;

a, n e m - parâmetros para ajuste da curva de retenção.

A difusividade do solo foi determinada segundo Klute (1965) e Pauletto et al. (1988), conforme a Equação 4, da seguinte forma:

$$
\mathrm{D}(\theta)=\mathrm{K}(\theta) \frac{\partial \mathrm{h}}{\partial \theta}
$$

O ajuste dos parâmetros das equações que representará o modelo para o solo foi realizado através do aplicativo RETC versão 6.02 (van GENUCHTEN et al., 2009): foi considerado o conteúdo de água, capacidade hidráulica, condutividade hidráulica de solo não saturado, efetivo conteúdo de água e difusividade versus o logaritmo da carga de pressão. A verificação da qualidade do ajuste pelo próprio aplicativo RETC foi feita por meio do índice de regressão.

Os dados obtidos foram submetidos à análise de variância pelo teste $\mathrm{F}$ a nível de $1 \mathrm{e}$ $5 \%$ de probabilidade, as médias comparadas entre si pelo teste Tukey à $5 \%$ de probabilidade, utilizando software Sisvar 5.3 (FERREIRA, 2011).

\section{RESULTADOS E DISCUSSÃO}

A umidade residual, o $\alpha$ e $\mathrm{o} n$ não apresentaram diferença significativa em relação ao sistema de plantio, já a umidade de saturação $\left(\theta_{\mathrm{s}}\right)$ e a condutividade hidráulica do solo saturado $(\mathrm{Ks})$, demonstraram diferença significativa, apresentando maior valor de $\theta_{\mathrm{s}}$ em sistema de plantio convencional e de Ks em sistema de plantio direto (Tabela 1). 
Tabela 1. Parâmetros do modelo obtidos a partir do ajustamento dos dados, a partir dos testes de infiltração com o programa RETC.

\begin{tabular}{|c|c|c|c|c|c|c|c|c|c|c|c|}
\hline \multirow{2}{*}{ Sistemas } & \multicolumn{2}{|l|}{$\theta_{\mathrm{r}}$} & \multicolumn{2}{|c|}{$\theta_{\mathrm{s}}$} & \multicolumn{2}{|l|}{$\alpha$} & \multicolumn{2}{|l|}{$n$} & \multicolumn{2}{|c|}{$\mathrm{K}_{\mathrm{S}}$} & \multirow{2}{*}{$\frac{\mathrm{R}^{2}}{\%}$} \\
\hline & $\mathrm{cm}^{3} \mathrm{c}$ & & $\mathrm{cm}^{3} \mathrm{c}$ & & $\mathrm{cm}^{-}$ & & - & & $\mathrm{cm}$ & & \\
\hline Direto & 0,0797 & $\mathrm{a}$ & & $\mathrm{b}$ & 0,0919 & $\mathrm{a}$ & 2,7303 & $\mathrm{a}$ & 1,67 & $\mathrm{a}$ & 98,6 \\
\hline Convencional & 0,0772 & $\mathrm{a}$ & 0,7181 & a & & $\mathrm{a}$ & 2,7370 & $\mathrm{a}$ & 1,55 & $\mathrm{~b}$ & 98,1 \\
\hline
\end{tabular}

"Média com a mesma letra minúscula na coluna não indica diferença significativa entre os sistemas de plantio pelo teste Tukey, a $5 \%$ de probabilidade. $\theta_{\mathrm{s}}$ - teor de umidade volumétrico de saturação; $\theta_{\mathrm{r}}$ - teor de umidade volumétrico residual; $\alpha$, $\mathrm{n}$ - parâmetros para ajuste da curva de retenção; $\mathrm{k}_{\mathrm{s}}$ - condutividade hidráulica na condição saturada.

O $\mathrm{R}^{2}$ para o conteúdo de água $(\theta(\mathrm{h}))$ nos dois sistemas de plantio foram maiores que $98 \%$, indicando que apenas 1,4 e 1,9\% (plantio convencional e direto) das variações do conteúdo de água não são explicadas pela variação da carga hidráulica. A $\mathrm{K}_{\mathrm{S}}$ no sistema de plantio direto foi $7,2 \%$ maior do que a observada no sistema de plantio convencional. Estes resultados são justificados, pois segundo Uyeda (2009) os maiores valores de Ks são encontrados, geralmente, onde se tem um maior grau de estruturação, a qual pode ser alterado por processos de agregação das partículas e formação de macroporos, os quais dependem da textura e demais condições do solo, além das práticas culturais adotadas.

O sistema de plantio direto tende a apresentar um maior valor de porosidade em relação ao sistema de plantio convencional. Mesquita \& Moraes (2004), afirmaram, de um modo geral, que os maiores valores de Ks são encontrados juntamente com os maiores valores de porosidade.

Para Uyeda (2009), em um dado solo, Ks é tanto maior quanto maior sua umidade $(\theta \mathrm{s})$. $\mathrm{O}$ valor máximo de Ks ocorre quando o solo se encontra saturado $(\theta=\theta \mathrm{s})$. Porém nos resultados observados o conteúdo de água do solo na zona saturada em sistema de plantio convencional foi $9,7 \%$ superior à verificada no sistema de plantio direto, entretanto o ponto de inflexão da curva para o sistema de plantio convencional ocorreu em uma carga hidráulica menor do que a observada no sistema de plantio direto. Como o conteúdo de água (zona saturada) no plantio convencional é maior do que a do plantio direto, a zona de transição também é maior no sistema de plantio convencional, onde o conteúdo de água diminuiu de maneira menos expressiva com o aumento da carga hidráulica do que em sistema de plantio direto (Figura 1).

A)

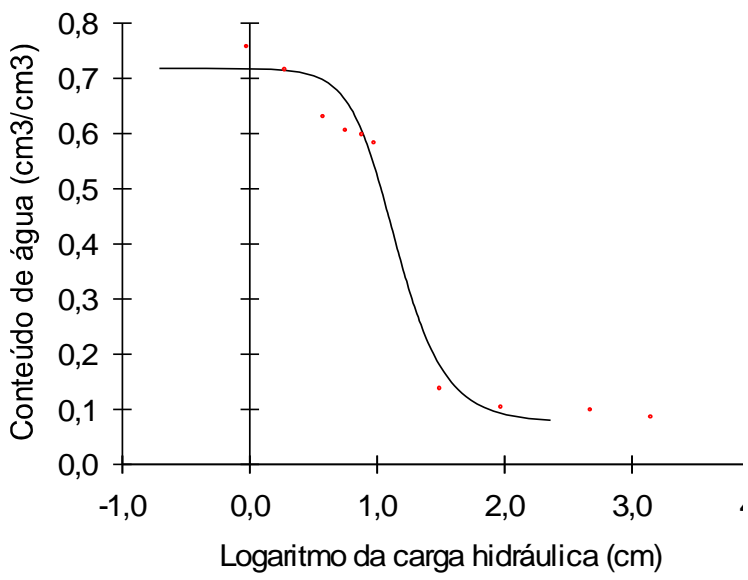

B)

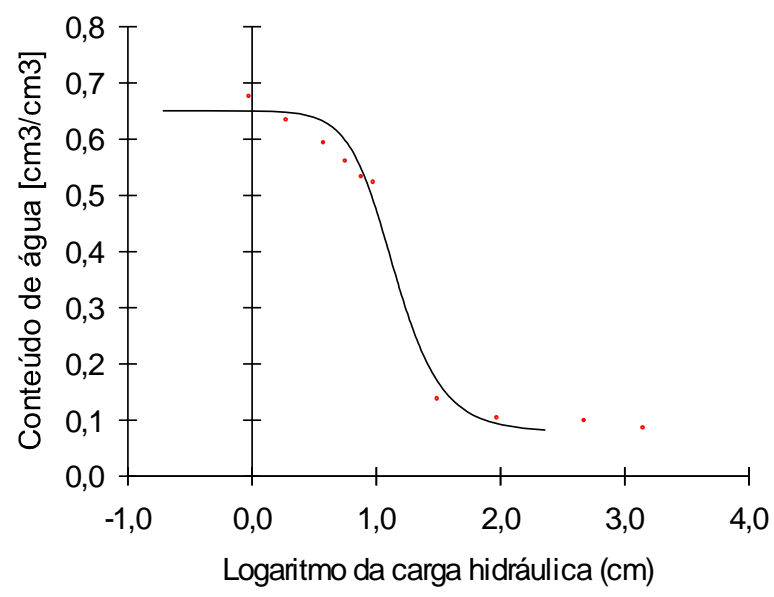

Figura 1. Conteúdo de água em função do logaritmo da carga hidráulica nos sistemas de plantio convencional (A) e direto (B). 
Elevados valores de $\theta$ s encontrados onde no sistema convencional onde ocorre uma mobilização e desestruturação do solo na camada superficial, presumivelmente refletem a importância da continuidade dos macroporos para fluxo de água na saturação, porém a explicação para isto não é clara, ou melhor, é contraposta ao que se entende sobre a influência da densidade do solo na natureza do sistema estrutural dos poros. Nas camadas superficiais, geralmente, há maior variação na densidade do solo devido ao manejo, o que pode propiciar a formação de poros com diâmetros maiores, os quais permitem maiores valores de condutividade hidráulica saturada, porém estes poros podem não influenciar muito a densidade do solo. A magnitude destas modificações estruturais ocasionadas pelo manejo depende da ocorrência e da freqüência

A)

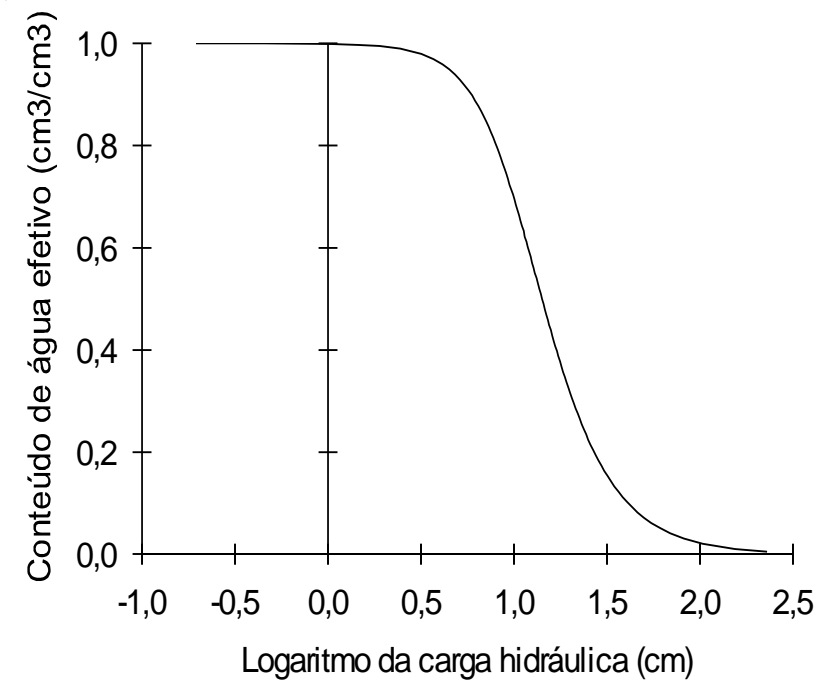

das operações de práticas agrícolas superficiais e subsuperficiais no solo e, também, do efeito compactante produzido pelo trânsito do maquinário agrícola ou de animais (MESQUITA \& MORAES, 2004), além disso, a morfologia, orientação e tamanho dos sistemas radiculares dos vegetais podem afetar a Ks.

O conteúdo de água efetivo foi equivalente para ambos os sistemas de plantio, isto ocorre devido o conteúdo de água variar de 0 a 1 , ou seja variar de $\theta_{\mathrm{r}}$ a $\theta_{\mathrm{s}}$; como foi verificado diferença significativa apenas no $\theta_{\mathrm{s}}$, o conteúdo de água efetivo torna-se restrito pelo $\theta_{\mathrm{r}}$ ocorrendo um efeito de afunilamento que reduz considerávelmente a diferença verificada no $\theta_{\mathrm{s}}$, sendo necessário, consequentemente, maiores diferenças para que se possa identificar variações relevantes no conteúdo de água efetivo (Figura 2).
B)

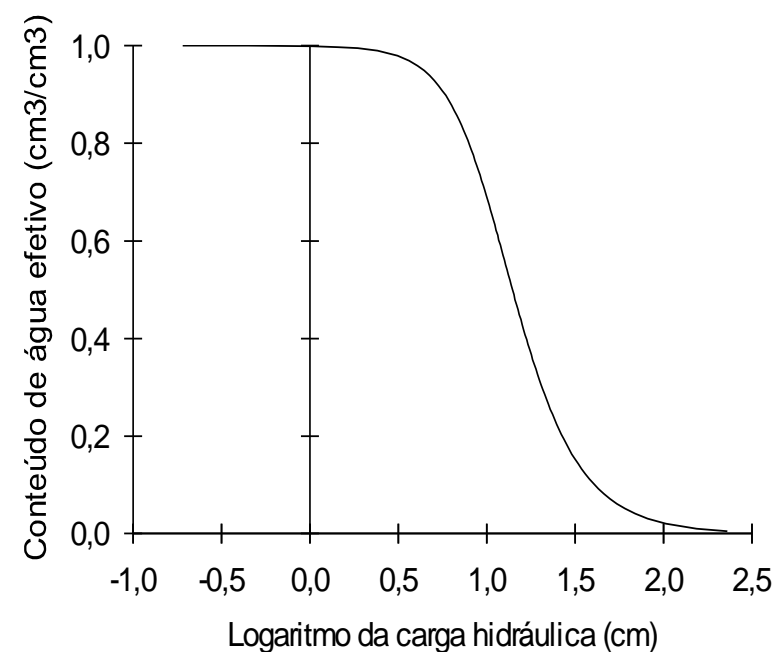

Figura 2. Conteúdo de água efetivo em função do logaritmo da carga hidráulica nos sistemas de plantio convencional (A) e direto (B).

A máxima capacidade hidráulica foi verificada no sistema de plantio convencional sendo esta $9,1 \%$ superior a encontrada no sistema plantio direto; esta maior capacidade hidráulica no sistema convencional deve-se ao fato da umidade de saturação ser mais alta neste sistema, logo existe uma área maior para o fluxo de água, o que implica diretamente em uma capacidade hidráulica mais elevada (Figura 3). 
A)

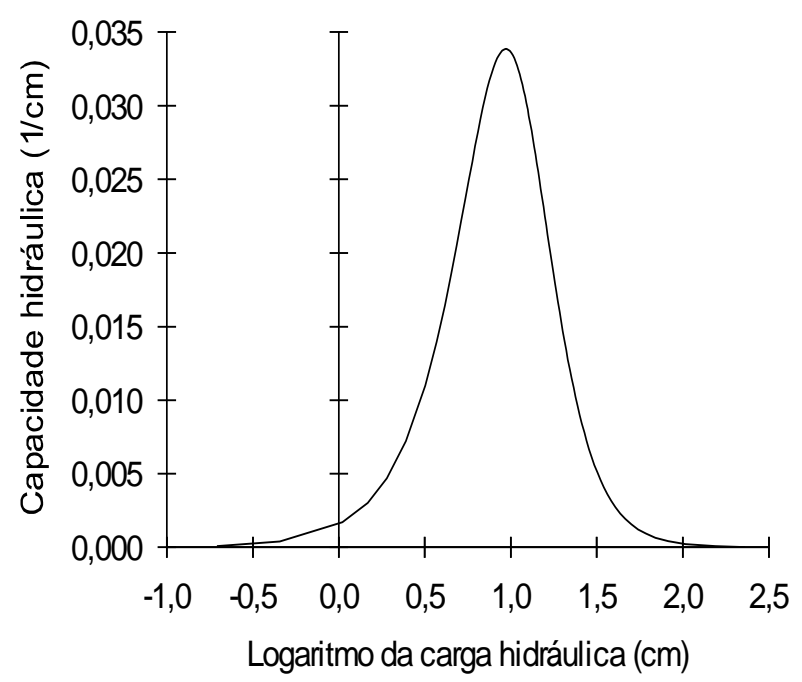

B)

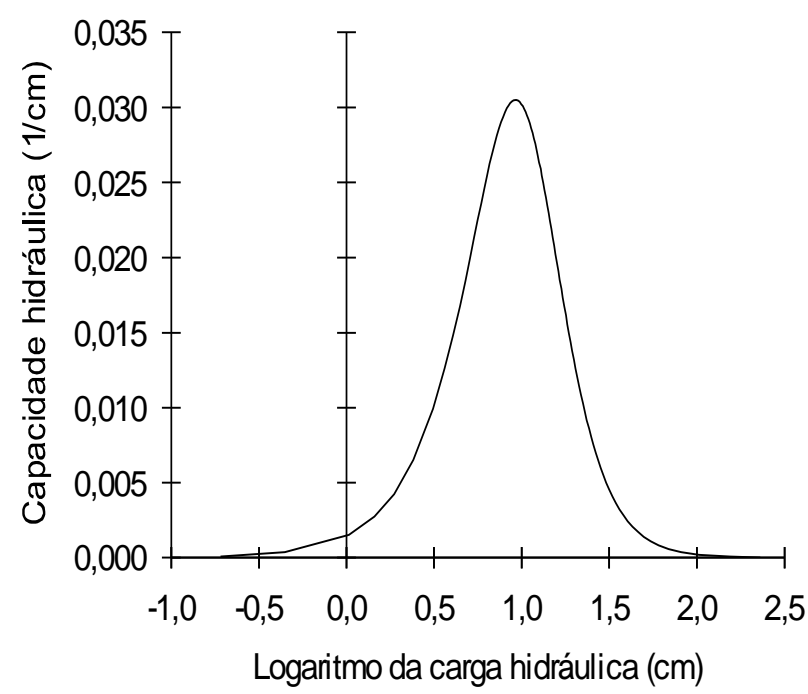

Figura 3. Capacidade hidráulica em função do logaritmo da carga hidráulica nos sistemas de plantio convencional (A) e direto (B).

A condutividade hidráulica do solo não saturado, na carga hidráulica de $0 \mathrm{~cm}$, no sistema de plantio direto se mostra 7,5\% acima da verificada em sistema de plantio convencional, de tal modo que o ponto de inflexão da curva para o sistema de plantio convencional ocorreu em uma carga hidráulica menor do que a observada no sistema de plantio direto. A metade da condutividade hidráulica do solo saturado ocorreu na carga hidráulica de 0,73 e $0,74 \mathrm{~cm}$ no sistema de plantio direto e convencional, respectivamente (Figura 4). Estes resultados estão de acordo com Silva et al. (2014), que afirmam que o manejo inadequado causa a compactação, redução da condutividade hidráulica do solo e consequentemente a redução na absorção de nutrientes e alteração na infiltração e redistribuição de água.
A)

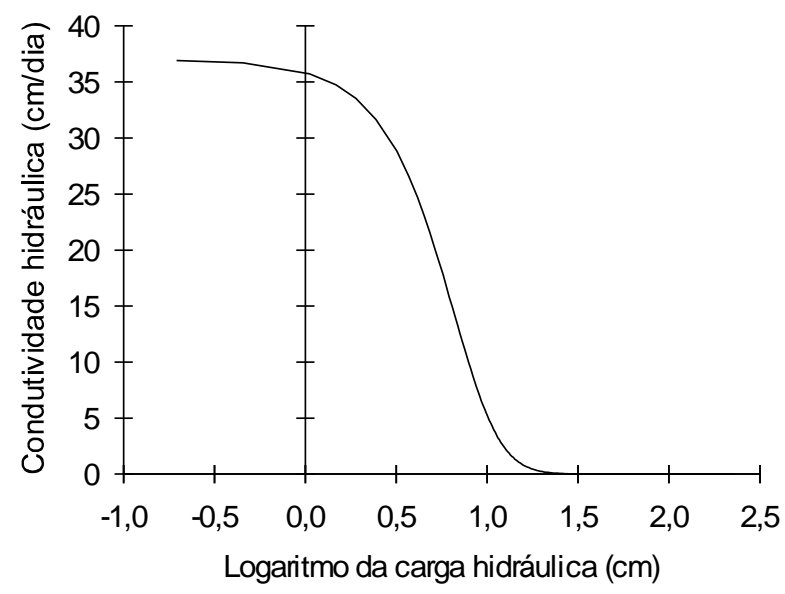

B)

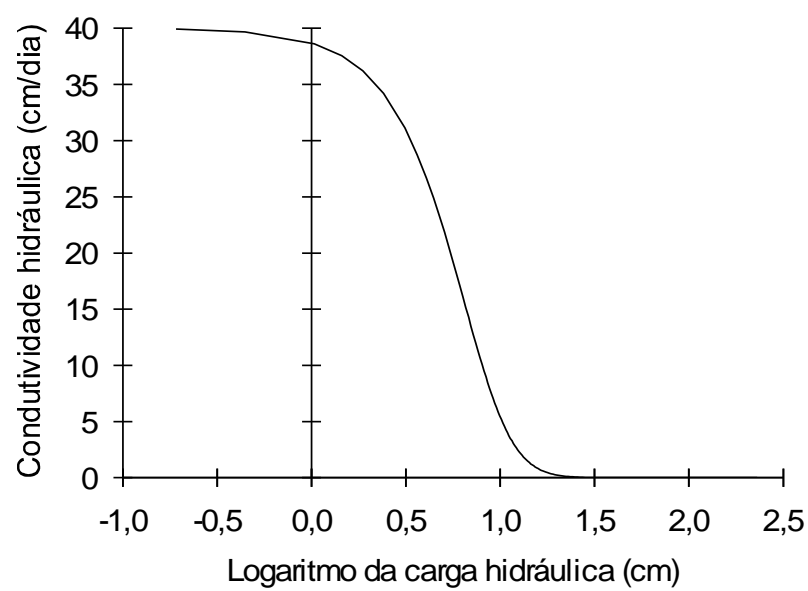

Figura 4. Condutividade hidráulica do solo não saturado em função do logaritmo da carga hidráulica em plantio convencional (A) e direto (B).

A condutividade hidráulica do solo saturado diferiu em função do sistema de plantio, e manteve essa diferença em cargas hidráulicas mais elevadas. A condutividade hidráulica do solo tende a diminuir com aumento da umidade, seu valor maximo ocorre na maxima umidade do solo, dessa forma, o sistema de plantio direto quando comparado ao 
convencional obteve os maiores valores. A condutividade hidráulica do solo é uma propriedade dinâmica, uma vez que o solo como meio poroso, pela sua formação, pode ter sua estrutura modificada por processos naturais, como é o caso do adensamento, e por processos antropogênicos (BRITO, 2010). Silva et al. (2014) observaram o impacto da agricultura nos solos do sudoeste goiano (Latossolo vermelho distroferrico), e demonstraram os estágios de degradação de solos sob diferentes condições de uso antrópico (Cerrado, Cana, Culturas Anuais e Integração LavouraPecuária-Floresta - ILPF), e evidenciam variabilidade dos atributos dos solos e a necessidade de pesquisas aplicadas de forma a obter limites referenciais de valores aceitáveis para aplicação nos diversos campos de estudo, e observaram

A)

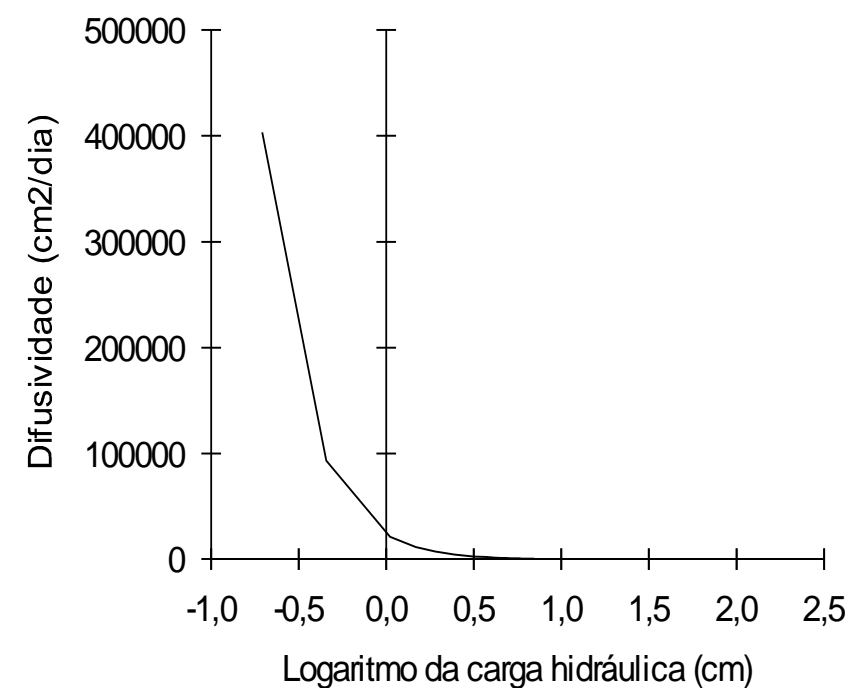

que Cerrado apresentou os maiores valores para Condutividade hidráulica do solo saturado, na profundidade estudada, diferindo estatisticamente dos demais sistemas de manejo. Os sistemas de cultivo Cana e ILPF mostraram valores intermediários, mas o sistema de Culturas anuais (plantio convencional) apresentou os menores valores para a avaliação em questão.

A difusividade hidráulica inicial no sistema de plantio direto foi $16 \%$ superior a difusividade do sistema de plantio convencional, essa diferença pouco se alterou com o aumento da carga hidráulica, de tal maneira que em cargas hidráulica mais elevadas (> $2 \mathrm{~cm})$, a diferença na difusividadade em função do sistema de plantio foi de aproximadamente $15,5 \%$ (Figura 5).

B)

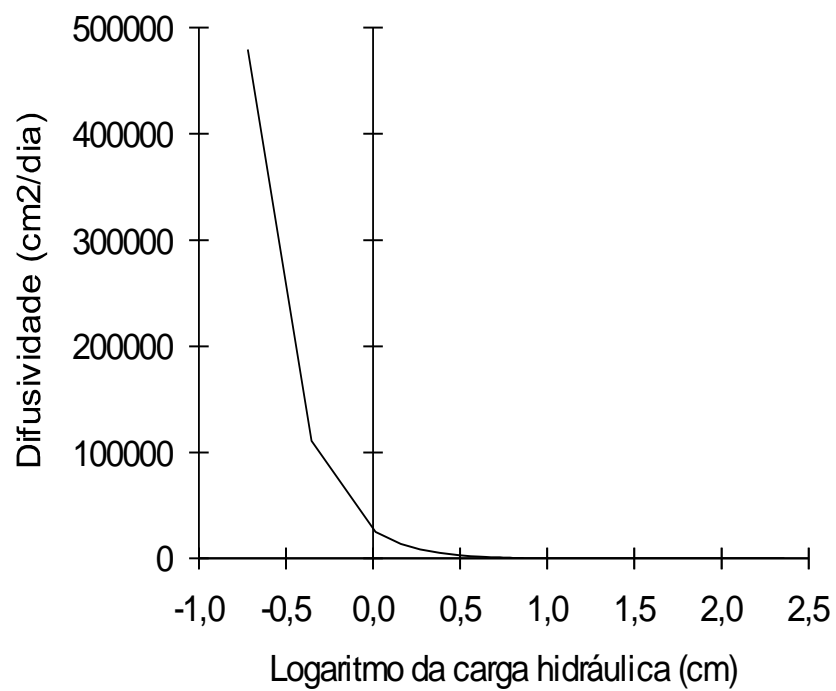

Figura 5. Difusividade hidráulica em função do logaritmo da carga hidráulica nos sistemas de plantio convencional (A) e direto (B).

O logaritmo da difusividade em função do logaritmo da carga hidráulica para ambos os sistemas de plantio apresenta um comportamento bem mais semelhante, devido a redução nas diferenças que foram encontradas na difusividade para os sistemas de plantio (Figura 6). 
A)

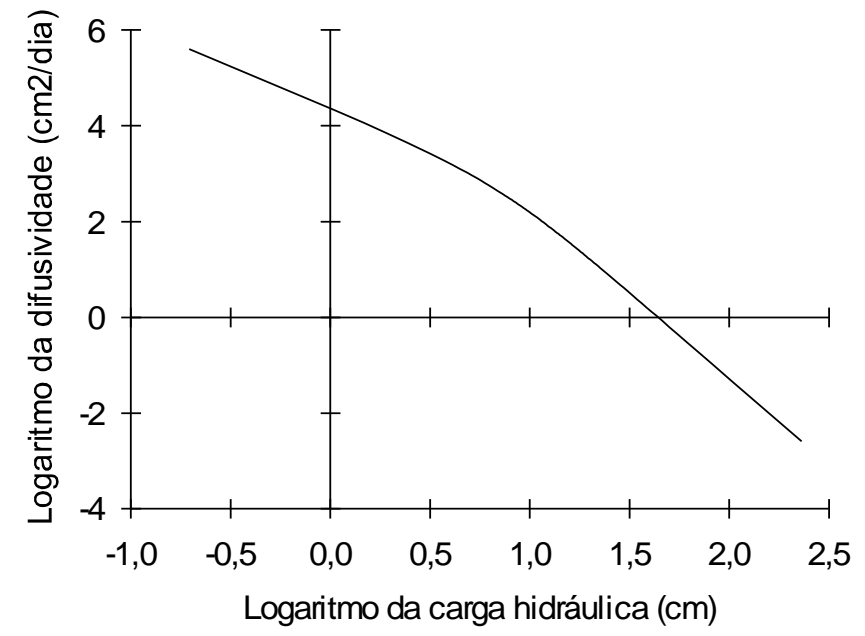

B)

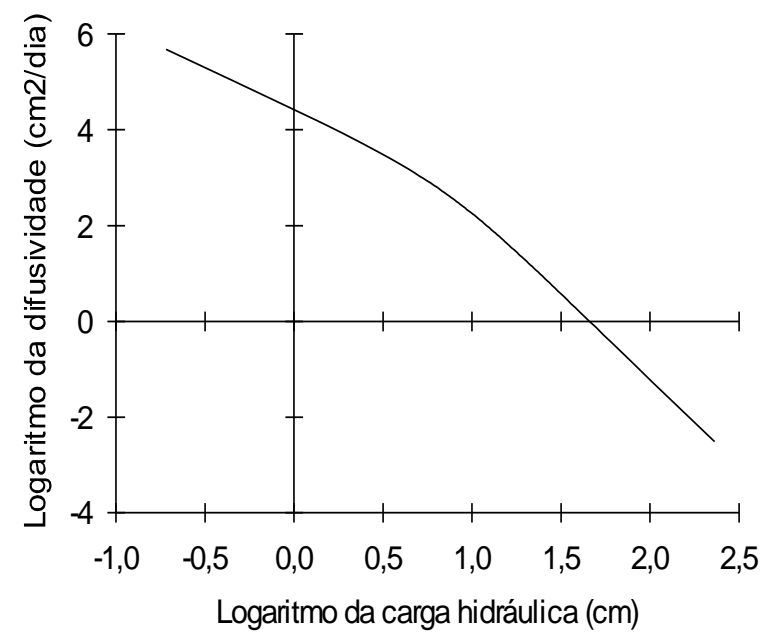

Figura 6. Logaritmo difusividade hidráulica em função do logaritmo da carga hidráulica nos sistemas de plantio convencional (A) e direto (B).

A carga hidráulica é igual a zero quando o logaritmo da difusividade é de 4,4 e $4,3 \mathrm{~cm}^{2}$ $\mathrm{dia}^{-1}$, sistema de plantio direto e convencional respectivamente, já o logaritmo da difusividade se torna igual a zero quando a carga hidráulica é de 1,66 e 1,64 cm. O logaritmo da difusividade inicial do sistema de plantio direto é $1,3 \%$ maior do que o do sistema convencional, e essa diferença é de aproximadamente $2,8 \%$ na carga hidráulica de $2,36 \mathrm{~cm}$. As classes de Latossolo apresenta as maiores vazões específicas e uma tendência de regularização de vazões (são essencialmente córregos perenes). A diminuição da condutividade observada com o aumento da profundidade é devida aos processos de translocação de argilas, pela redução da estruturação granular e grumosa (FIORI, 2010). Além da distribuição e tamanho dos poros, a tortuosidade e a conectividade dos poros são as características da geometria do espaço poroso que mais influenciam no transporte de fluidos no solo (CHIEF et al., 2006; BRITO, 2010).

As práticas de preparo do solo comumente utilizadas tendem a proporcionar uma maior uniformidade quanto aos aspectos físicos do solo; o conteúdo de água na camada de 0-
0,15 m é mais variável no sistema de plantio direto, enquanto que em sistema de plantio convencional (escarificação entre 0,4 e $0,5 \mathrm{~m} \mathrm{e}$ gradagem profunda) há maior dependência espacial; a maior homogeneidade da área com sistema de plantio convencional se dá pelo fato de a aração e a gradagem, enquanto no plantio direto a atuação de processos biológicos é mais predominante e aleatório no espaço, causando uma maior variabilidade (ZANETTE et al., 2007; SCHAFFRATH et. al., 2008).

A importância de Ks pode ser justificada, então, pela sua participação na determinação do fluxo de água nos solos e, do ponto de vista prático, para tomada de decisão sobre projetos de irrigação, drenagem, quantificação da erosão, lixiviação de substâncias químicas, com consequências na poluição e contaminação de camadas mais profundas do solo e até mesmo do lençol freático. Assim, a Ks tem influência marcante nestas e na maioria de outras práticas de manejo do solo. Esses processos são importantes para o adequado manejo do solo e da cultura (BRITO et al., 2009; GHIBERTO et al., 2009), da irrigação e no monitoramento de uma possível poluição do lençol freático. 


\section{CONCLUSÃO}

Os maiores valores de conteúdo de água no solo e capacidade hidráulica foram observados no sistema de plantio convencional, porém os maiores valores de condutividade hidráulica e difusividade hidráulica inicial no sistema de plantio direto superior ao sistema de plantio conveniconal, essa diferença pouco se alterou com o aumento da carga hidráulica.

O sistema plantio direto tende a continuar com uma condutividade hidráulica em condição saturada maior do que a observada no sistema de plantio convencional, contribuindo para uma menor escoamento superficial e/ou sucetibilidade a erosão.

\section{AGRADECIMENTOS}

Os autores agradecem ao Ministério da Ciência e Tecnologia (MCT), ao Conselho Nacional de Desenvolvimento Científico e Tecnológico $(\mathrm{CNPq})$ e à Coordenação de Aperfeiçoamento de Pessoal de Nível Superior (Capes).

\section{REFERÊNCIAS BIBLIOGRÁFICAS}

BERNARDO, S; SOARES, A. A.; MANTOVANI, E. C. Manual de irrigação. 8. Ed. Atual. e Ampl. Viçosa: UFV, 2006. 625p.

BRITO, A. S. Variabilidade espacial da condutividade hidráulica e da permeabilidade ao ar em função dos conteúdos de água e ar no solo. Tese (Doutorado) - Escola Superior de Agricultura “Luiz de Queiroz”, Piracicaba, 2010.

BRITO, A. S.; LIBARDI, P. L.; GHIBERTO, P. J. Water Balance Components in Soils Cropped to Sugarcane, with and without Nitrogen Fertilization. Revista Brasileira de Ciência do Solo, v. 33, n. 2, p. 295-303, 2009.

CHIEF, K.; FERRÉ, T. P. A.; NIJSSEN, B. Fiel $\mathrm{d}$ testing of a soil corer air permeameter
(SCAP) in desert soils. Vadose Zone Journal, Madison, v. 5, n. 4, p.1257-1263, Nov., 2006.

CONCEIÇÃO, B. S. Dinâmica de água e potássio em substratos comerciais. Dissertação (Mestrado). 2013. 92 f. Universidade Federal de Lavras, Minas Gerais, 2013.

CUNHA, J. L. X. L.; ALBUQUERQUE, A. W.; SILVA, C. A.; ARAÚJO, E. de; SANTOS JUNIOR, R. B. dos. Velocidade de infiltração da água em um latossolo amarelo submetido ao sistema de manejo plantio direto. Revista Caatinga, v.22, n.1, p.199-205, 2009.

FEDDES, R. A.; KOWALIK, P; KOLINSKAMALINKA, K.; ZARADNY, $\mathrm{H}$. Simulation of field water uptake plants using a soil water dependent root extraction function. Journal of Hydrology, Amsterdam, v.31, p.13-26, 1976.

FERREIRA, D. F. Sisvar: a computer statistical analysis system. Ciência e Agrotecnologia, v. 35, n.6, p. 1039-1042, 2011.

FIORI, J. P. O.; CAMPOS, J. E. G.; ALMEIDA, L. Variabilidade da condutividade hidráulica das principais classes de solos do estado de goiás. Geociência (São Paulo). v.29, n.2, p. 229-235, 2010.

GHIBERTO, P.J.; LIBARDI, P.L.; BRITO, A.S.; TRIVELIN, P.C.O. Leaching of nutrients from a sugarcane crop growing on an Ultisol in Brazil. Agricultural Water Management, Amsterdam, v. 96, p. 1443-1448, 2009.

GONÇALVES, A. D. M. A.; LIBARDI, P. L. Análise da determinação da condutividade hidráulica no solo pelo método do perfil instantâneo. Revista Brasileira de Ciência do Solo, v.37, p.1174-1184, 2013.

KLUTE, A. Laboratory measurement of hydraulic conductivity of saturated soil. In: BLACK, C. A. (Ed.). Methods of soil analysis: part 1. Madison: American Society of Agronomy, p. 210-221. 1965. 
LAWLOR, D. W., CORNIC, G. Photosynthetic carbon assimilation and associated metabolism in relation to water deficits in higher plants. Plant, Cell \& Environment, v.25, p.275-294, 2002.

MELO, R. M.; BARROS, M. F. C. Efeito da qualidade de água de irrigação na condutividade hidráulica de solos de Pernambuco. Periódico anual técnicocientífico do CEFET-RR. Norte Científico, v.2, n.1, 2007.

MESQUITA, M. G. B. F.; MORAES, S. O. A dependência entre a condutividade hidráulica saturada e atributos físicos do solo. Ciência Rural, Santa Maria, v.34, n.3, p.963-969, 2004.

MUALEM, Y. A new model for predicting the hydraulic conductivity of unsaturated porous media. Water Resource Research, v.12, p.513-522, 1976.

PAULETTO, E.A.; LIBARDI, P.L.; MANFRON, P.A. \& MORAES, S.O. Determinação da condutividade hidráulica a partir da curva de retenção de água. Revista Brasileira de Ciência do Solo, Viçosa, MG, v. 12, p. 189-195, 1988.

REICHARDT, K.; TIMM, L. C. Solo, planta e atmosfera: conceitos, processos e aplicações. 2.ed. Barueri: Manole, 2012. 524 p.

SCHAFFRATH, V.R.; TORMENA, C.A.; FIDALSHI, J.; GONÇALVES, A.C.A. Variabilidade e correlação espacial de propriedades físicas de solo sob plantio direto e preparo convencional. Revista Brasileira de Ciência do Solo, Viçosa, v. 32, p.1369-1377, 2008.

SILVA, A. L. de. Variabilidade dos componentes do balanço hídrico: um estudo de caso em uma cultura do cafeeiro (Coffea arábica L.) no Brasil. 2005. 72f. Tese (Doutorado em Agronomia). Escola Superior de Agricultura "Luiz de Queiroz", Universidade de São Paulo, Piracicaba, 2005.
SILVA, A. A. M.; AZEVEDO, L. P.; SAAD, J. C. C.; MICHELS, R. N. Propriedades físicohídricas, desenvolvimento radicular e produtividade da soja em dois tipos de manejos de solo. Irriga, Botucatu, v.17, n.3, p.387-396, 2012.

SILVA, N. F.; CUNHA, F. N.; OLIVEIRA, R. C.; CABRAL FILHO, F. R.; TEIXEIRA, M. B.; CARVALHO, J. J. Características físicohídricas de um Latossolo sob diferentes sistemas de manejo. Revista Brasileira de Agricultura Irrigada, v.8, $\mathrm{n}^{\mathrm{o}}$. 5, p.375 - 390, 2014.

SOBRINHO, T.A.; VITORINO, A.C.T.; SOUZA, L.C.F. de.; GONÇALVES, M.C.; CARVALHO, D.F. de. Infiltração de água no solo em sistemas de plantio direto e convencional. Revista Brasileira de Engenharia Agrícola e Ambiental, Campina Grande, v.7, n.2, p.191-196, 2003.

UYEDA, C. A. Influência da aplicação de vinhaça na condutividade hidráulica do solo saturado e no escoamento superficial. 66p. 2009. Tese (Doutorado) - Escola Superior de Agricultura "Luiz de Queiroz", 2009.

van GENUCHTEN M. T.; LEIJ, F. J.; YATES, S. R. RETEC, Code for quantifying the hydraulic functions of unsaturated soils: version 6.02. Riverside: University of California, 2009.

van GENUCHTEN, M. Th. A closed-from equation for predicting the conductivity of unsaturated soils. Soil Science Society of American Journal, Madison, v.44, p.892-898, 1980.

ZANETTE, S.V.; SILVESTRE, M.G.; BOAS, M.A.V.; UIRBE-OPAZO, M.A.; QUEIROZ, M.M.F. Spatial analysis of soil moisture with soybean under two management systems. Revista Brasileira de Engenharia Agrícola e Ambiental, Campina Grande, v. 11, n. 3, p. 239-247, 2007. 